\title{
The "Perfect" Latte, According to a Benumbed Barista
}

\author{
Andrea Mohler
}

7-9 grams per shot

$12 \mathrm{oz}$

5 pumps

$21 / 2$ pumps
Arabica Beans

Milk of any variety

Flavoring syrup

or

Flavor sauce of choice

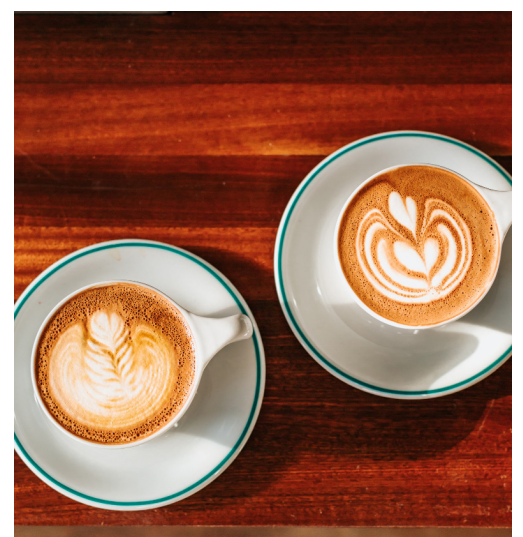

Photo by Tyler Nix on Unsplash

Description: two mugs with frothed milk on a counter top.

Pour at least 15 grams of the arabica beans into a coffee grinder and grind until the beans are reduced to a smooth, sand-like texture.

Deposit the grounds into a portafilter.

Use a tamper to press the grounds to a level surface. Use exactly 25 lbs. of pressure.

Lock the portafilter into the filterhead, and set two shot glasses underneath the filterhead.

Flip on the filterhead and mop up the spill beneath the third filterhead on the right.

Remind your coworker to let Jim know the machine is leaking again.

Wait exactly 27 seconds after pulling the shot to flip the head off. Anywhere between 25 and 30 seconds should be viable for a shot. The machine is old, though, and sometimes that number is off. And sometimes it's humid, which creates another variable. And sometimes the customer is an asshole, which means the shots do not have to be perfect. A lot of things can throw off the timing for shots.

While the shot is pulling, fill your cup with $2 \frac{1}{2}$ pumps of flavored sauce or five pumps of flavored syrup. While this number seems excessive, the syrup is about the only way the general public can find a latte palatable. Don't question the proportions. 


\section{Recipe}

Pour approximately $300 \mathrm{ml}$ of whatever milk you're using into the steaming cup.

Begin frothing the milk:

Hold the cup at the bottom of the cup until you start to feel a small amount of warmth at the bottom of the cup.

Pull the cup downward so the frothing wand skims the surface of the milk.

Pull the cup back up again so the frothing wand is submerged.

Slowly repeat the process until the frothing wand atoms are screaming at you.

Tap the cup against the counter to knock out any bubbles.

Have a customer yell at you to not make their latte too hot; they burnt their tongue last time.

Dump out the milk and repeat the process, this time only allowing the milk to get to "kid's

temperature."

Pour the espresso shots over the syrup/sauce in the cup and mix together until homogenous.

Begin pouring the milk. Do not waste time with a fancy design, they do not check and they do not care. Place the lid and the sleeve onto the cup and call out the flavor of the latte, setting it down on the outgoing counter.

Get tipped 50 cents. If you're lucky. 